\title{
CINQ CAS DE FIẼVRE BOUTONNEUSE MÉdITERRANÉENNE EN SUISSE
}

\author{
E. CHAMOT*, P. CHATELANAT**, L. HUMAIR*,
}

A. AESCHLIMANN***, J. BOWESSIDJAOU***

RÉSUMÉ. La Fièvre Boutonneuse Méditerranéenne (FBM), également appelée Fièvre de Marseille ou Fièvre Eruptive Méditerranéenne, est une maladie commune en Afrique Noire, aux Indes et dans les pays méditerranéens, comme son nom l'indique. Rickettsia conorii en est l'agent et Rhipicephalus sanguineus le vecteur principal. En Suisse, cette tique ne peut survivre que dans nos habitations où elle est introduite par un chien ayant accompagné son maître en voyage. Pour cette raison, la maladie est rare dans notre pays et ne s'observe que chez des personnes rentrant de vacances ou chez des patients en contact avec des Rhipicéphales importés. Dans cet article, nous rapportons cependant cinq cas de FBM survenus en Suisse Romande entre 1980 et 1985. Nous insistons particulièrement sur les relations épidémiologiques complexes existant entre quatre de ces cas. Nous présentons encore la clinique de cette affection et nous passons en revue les modalités de son diagnostic, de son traitement et du dépistage d'éventuels foyers domestiques de $R h$. sanguineus.

Mots-clés : Tique (Rhipicephalus sanguineus). Rickettsie (Rickettsia conorii). Importation en Suisse.

\section{On five cases of Mediterranean boutonneuse fever in Switzerland.}

SUMMARY. The Boutonneuse Fever also called Marseilles or Eruptive Mediterranean Fever is an common disease in mediterranean area, in Africa and Indien. Rickettsia conorii is the microorganism involved and Rhipicephalus sanguineus is the most common vector.

In Switzerland, this dog's tick don't exist in the nature, but it may be introduced by a dog contaminated elsewhere outside of the country and remain alive in our houses. It the reason why this disease is very uncommon in Switzerland. However, it may be observed in people coming back from vacation or in patients in contact with imported Rhipicephalus.

In this paper, five cases of Boutonneuse Fever diagnosed between 1980 and 1985 are reported. The epidemiological relationships between four of these cases are of special interest. The clinical picture of the disease is presented with the differents serological tests useful in confirming the diagnosis. Prevention and treatment are discussed.

Key-words: Tick (Rhipicephalus sanguineus). Rickettsia (Rickettsia conorii). Importation in Switzerland.

* Service de Médecine, Hôpital Communal, CH-2300 La Chaux-de-Fonds.

** Groupe médical de Moillebeau, rue de Moillebeau 33, CH-1209 Genève.

** Institut de Zoologie, Chantemerle 22, CH-2000 Neuchâtel.

Travail effectué en partie avec l'aide du Fonds national suisse de la recherche scientifique.

Accepté le 24 février 1987. 


\section{Introduction}

Le développement considérable des voyages intercontinentaux, souvent effectués avec des animaux domestiques, a entraîné l'apparition dans nos pays de nombreuses maladies exotiques, parasitaires en particulier. Si celles-ci peuvent être redoutables, elles sont en général méconnues, non seulement du public, mais également de beaucoup de médecins et de vétérinaires.

La Fièvre Boutonneuse Méditerranéenne (FBM), due à Rickettsia conorii, fait partie de ces affections. Son vecteur principal est Rhipicephalus sanguineus, une tique commune dans les régions tropicales et méditerranéennes. Elle est fréquemment importée dans le centre et le nord de l'Europe occidentale $(1,3,10,12,23)$ par des chiens de vacanciers au retour de voyages. Si elle ne survit pas dans la nature sous nos latitudes, elle s'adapte, en revanche, très bien au microclimat rencontré dans nos habitations.

\section{CLINIQUE DE LA FIËVRE BOUTONNEUSE MÉDITERRANÉENNE (FBM)}

La FBM s'observe en Europe entre les mois de juin et d'octobre (21) (85\% des cas entre juillet et septembre pour Raoult (18)). Cette maladie survient à tout âge et touche aussi bien les femmes que les hommes. Une anamnèse de piqûre de tique est retrouvée dans $10 \%$ des cas (18), tandis que la présence d'un chien dans l'entourage est notée chez 30 à $80 \%$ des patients $(15,19)$.

L'infection évolue en 4 phases. Premièrement une phase d'incubation dont la durée est variable mais se situe généralement entre 4 et 15 jours $(15,18,21)$. Elle est suivie d'une phase pré-éruptive qui débute souvent brutalement par l'apparition d'un état fébrile avoisinant les $39,5^{\circ} \mathrm{C}$. II s'accompagne d'un syndrome algique comprenant des céphalées tenaces et des myalgies parfois très intenses dans environ $1 / 3$ des cas. Les arthralgies sont plus rares (18).

La phase éruptive se caractérise par la triade Tache noire de Pieri-État fébrileExanthème.

La " tache noire " est pathognomonique. Il s'agit d'une escarre noirâtre de quelques millimètres de diamètre qui se développe au lieu de piqûre dans 40 à $85 \%$ des cas $(4,9,15,18)$ durant la phase éruptive ou en fin de phase pré-éruptive (18). Le plus souvent indolore, elle est entourée d'un halo érythémateux et parfois de quelques adénopathies satellites. Souvent difficile à mettre en évidence, elle siège surtout dans les grands plis, sur le tronc ou les membres inférieurs, parfois à la tête, principalement chez les enfants $(15,18,21)$. Elle peut être remplacée dans environ $5 \%$ des cas par une conjonctivite unilatérale $(4,9,16,18)$, témoignant du passage transconjonctival des rickettsies.

La fièvre, en plateau, élevée, décroît progressivement en 1 à 3 semaines en l'absence de traitement $(15,18,21)$. Elle s'accompagne d'une dissociation du pouls, relativement lent, dans la moitié des cas environ $(4,18,19)$ et parfois d'une splénomégalie chez l'enfant $(9,18)$. 
L'exanthème marque le début de la phase d'état (19). Rarement absent, il survient 1 à 7 jours après les premiers symptômes $(15,21)$. Il peut apparaître en tout point du corps, mais le plus souvent sur les membres, au voisinage des grosses articulations (21). Il se généralise en 1 à 3 jours $(4,18)$, pour toucher, finalement, les paumes des mains, les plantes des pieds et même le visage dans la moitié des cas $(18,19)$. Les éléments sont généralement maculopapuleux, parfois nodulaires et leur couleur varie du rose pâle au pourpre. Ils peuvent être purpuriques dans les formes graves $(4,18,21)$. Leur nombre varie de quelques-uns à quelques dizaines (19). Au cours de leur évolution, ils s'aplatissent progressivement et s'entourent d'une fine desquamation avant de disparaître ou, parfois, de laisser place à une très discrète hyperpigmentation résiduelle (16). Plusieurs poussées successives sont possibles (21).

Cette phase éruptive est suivie d'une période de convalescence, caractérisée par une asthénie souvent de longue durée (7).

Bien que généralement bénigne, même en l'absence de traitement, la FBM peut se compliquer :

1 - D’un syndrome méningé dans 10 à $15 \%$ des cas $(18,21)$. Généralement peu marqué, il peut apparaître avant, le plus souvent pendant ou après la phase éruptive. Il associe des céphalées, des vomissements et une petite raideur de nuque. Le LCR est celui d'une méningite à liquide clair, avec une pléiocytose à mononucléés et une protéinorachie modérée, tandis que la pression, le glucose et le chlore sont normaux. Des atteintes neurologiques périphériques peuvent s'observer (méningoradiculite, paralysie de nerfs crâniens...) (20).

2 - D'un syndrome encéphalitique (environ $10 \%$ des cas) $(18,20$ ) qui se manifeste surtout par une diminution de la vigilence et des perturbations électroencéphalographiques non spécifiques, parfois par des convulsions ou un syndrome cérébelleux.

3 - D'une atteinte cardiaque (environ $10 \%$ des cas) (18) qui se traduit par des extrasystoles, une bradycardie sinusale, un bloc de branche, une péricardite ou un collapsus.

4 - D'une atteinte digestive (environ 208 des cas) $(11,18,19)$ soit sous forme d'une atteinte hépatique avec cytolyse modérée, associée parfois à une hépatomégalie sensible et à un ictère mixte, soit, chez l'enfant, sous forme d'une atteinte entéritique avec douleurs abdominales, vomissements et diarrhées.

5 - D'une atteinte pulmonaire (dans environ $8 \%$ des cas) $(18,19)$, se traduisant par de la toux, des râles bronchiques et parfois des images réticulonodulaires sur la radiographie thoracique.

6 - D'une atteinte rénale (environ $5 \%$ des cas) (18), rarement cliniquement manifeste mais importante pour le pronostic.

7 - D'une atteinte articulaire (18), peu fréquente, sous forme d'arthrites séreuses. 
8 - D'une atteinte oculaire : uvéite ou choriorétinite, rare mais classique $(18,20)$.

Les formes malignes associent des atteintes encéphalitiques, rénales, hématologiques et hépatiques. Elles s'observent dans $6 \%$ des cas et touchent surtout les personnes âgées, les immunodéprimés et les personnes atteintes d'un déficit en G6PD (21). Malgré un traitement antibiotique précoce, le décès survient encore dans 1 à $2 \%$ des cas (21).

\section{EXAMENS PARACLINIQUES}

La vitesse de sédimentation est le plus souvent légèrement augmentée (18). L'anémie ne s'observe guère que chez l'enfant (18) et l'on a décrit aussi bien une leucopénie (surtout au début de la maladie), qu'une leucocytose à polynucléaires neutrophiles ou même parfois un syndrome mononucléosique $(18,21,22)$. Les thrombopénies sévères sont un indice de gravité.

L'hyponatrémie est fréquente (50\% des cas) tout comme l'hypokaliémie (25\% des cas) (18). Une élévation des transaminases s'observe en cas d'atteinte hépatique $(11,18)$, touchant davantage les GOT que les GPT (4). La phosphatase alcaline et la bilirubine sont plus inconstamment perturbées. On note parfois la présence d'une albuminurie, d'une microhématurie et d'une ascension transitoire de la créatininémie $(4,18,21)$.

Plusieurs tests sérologiques ont été mis au point au cours des 30 dernières années. La classique réaction de Weil-Felix correspond à l'agglutination par le sérum du patient de souches de Proteus ayant des antigènes communs avec $R$. conorii (souches OX2, OX19 ou OXK2). Peu spécifique, ne se positivant que tardivement durant la phase de convalescence, elle est pratiquement abandonnée (21). De même, la réaction de fixation du complément est en voie d'être remplacée par le test d'immunoffuorescence indirecte, plus fiable. Avec ces techniques, on observe l'apparition d'IgM spécifiques vers le $10^{\mathrm{e}}$ jour de la maladie. Les IgG se positivent plus tardivement mais peuvent rester élevés plus de 4 ans après une FBM. La réapparition d'IgM suggère une réinfection (14). Des titres supérieurs ou égaux à $1 / 20$ ou $1 / 40$ sont généralement considérés comme spécifiques $(6,8,13,18,21)$. Dans les formes graves, une biopsie de la tache noire permet parfois d'obtenir le diagnostic avant l'apparition de l'éruption (21).

\section{TRAITEMENT}

Les antibiotiques de premiers choix sont les tétracyclines (doxycycline 200$300 \mathrm{mg} /$ jour pendant environ 10 jours). Après leur administration la fièvre disparaît généralement en 2 ou 3 jours tout comme l'éruption. Lorsque les tétracyclines sont contre-indiquées, on les remplace par le chloramphénicol ou la spiramycine. Les pénicillines et le cotrimoxazol sont inefficaces $(15,18,21)$.

Dans cet article, nous rapportons 4 cas de FBM qui se s int déclarés dans une même famille suisse. Nous verrons qu'il existe des relations épidémiologiques 
complexes entre ces 4 cas, l'un d'entre eux ayant été observé à une centaine de kilomètres des trois autres. Nous exposons encore un $5^{\mathrm{e}}$ cas, cliniquement patent, mais dont le mode de contamination a été difficile à préciser, montrant ainsi l'importance d'une enquête épidémiologique minutieuse.

\section{DESCRIPTION DES CAS}

Cas no 1. Le 15 septembre 1980, une jeune femme de 35 ans, domiciliée dans la campagne genevoise, en bonne santé habituelle, développe un état fébrile à $40^{\circ} \mathrm{C}$ avec d'importantes myalgies, des arthralgies et des céphalées diffuses. Elle reçoit tout d'abord des salicylés durant $48 \mathrm{~h}$, puis $3 \times 500 \mathrm{mg}$ de Pivampicilline. Au $5^{\mathrm{e}}$ jour, on ne note aucune amélioration de son état et elle est de plus en plus abattue. L'examen somatique ne révèle rien de particulier, si ce n'est une petite lésion de $5 \mathrm{~mm}$, quelques centimètres au-dessus de la ligne inguinale gauche, ulcérée, avec un centre noir, accompagnée d'une adénopathie satellite inguinale gauche douloureuse. La formule sanguine est sans particularité (8500 leucocytes avec une répartition normale), la VS de $20 \mathrm{~mm}$ / première heure, les tests hépatiques sont normaux, les sérologies conventionnelles pour les virus et les rickettsies négatives, la radiographie pulmonaire normale.

$\mathrm{Au} 6^{\mathrm{e}}$ jour, on note l'apparition d'une éruption maculopapulaire discrète mais généralisée sur les membres et le tronc, non prurigineuse. L'hypothèse d'une rickettsiose est alors émise, entretenue par le fait que le chien de la patiente avait arboré des tiques récemment. Un traitement de tétracyclines, $4 \times 500 \mathrm{mg} /$ jour, est alors instauré. Il amène une amélioration spectaculaire de l'état clinique 24 heures plus tard et la disparition complète de la symptomatologie en 4 jours, sans séquelles.

L'examen sérologique (microagglutination) pratiqué 1 an $1 / 2$ plus tard contre Rickettsia conorii montre un titre à $1 / 256$ (Péter et al., 1984).

Cas nos 2 et 3. En juillet 1981, l'appartement de la patiente n० 1 est occupé durant son absence (et celle du chien) par un couple et leurs deux enfants âgés de 2 et 4 ans. Une dizaine de jours après leur arrivée, les deux enfants développent, presque simultanément, un état fébrile à $40^{\circ} \mathrm{C}$, avec prostration, arthralgies, myalgies intenses et céphalées, suivi cinq jours plus tard par une éruption généralisée décrite comme de type "varicelliforme "mais sans vésicule. On note également chez les deux enfants, dans la région du cou, une lésion différente, s'ulcérant après quelques jours avec un centre noir. I] n'y a pas de signes méningés, ni d'adénopathie. La formule sanguine est pratiquement la mème dans les deux cas, avec 10000 leucocytes et une déviation gauche $(18,5 \%$ de non segmentés, $37 \%$ de segmentés, $0 \%$ d'éosinophile, $2,5 \%$ de monocytes et $43 \%$ de lymphocytes). L'évolution étant en défaveur d'une varicelle ou d'une autre virose, l'hypothèse d'une rickettsiose est alors évoquée à la lumière du cas no 1 . Un traitement de chloramphénicol $50 \mathrm{mg}$ $\mathrm{kg} /$ jour est alors instauré avec un résultat spectaculaire. Deux jours plus tard, les enfants sont afébriles et le traitement est poursuivi pendant une semaine à la dose de $25 \mathrm{mg} / \mathrm{kg} / \mathrm{jour}$, amenant une guérison complète.

Cas $n^{\circ}$ 4. Le 16 septembre 1981, une dame de 77 ans, en excellente santé habituelle, vivant dans la campagne neuchâteloise et qui avait gardé le chien de la patiente $n^{\circ} 1$ en juillet 1981 , développe, à son tour, un état fébrile à $40^{\circ} \mathrm{C}$ avec arthralgies, myalgies, céphalées diffuses et intenses. Elle est mise aux salicylés, mais, trois jours plus tard, le tableau clinique s'est péjoré avec un état confusionnel, une faiblesse extrême, des myoclonies du membre supérieur droit et de la mâchoire, sans méningisme. On note surtout à ce moment-là une éruption maculopapuleuse généralisée, non prurigineuse, ainsi qu'une lésion ulcéreuse à centre noir avec une auréole rosée entre l'ombilic et le pli inguinal drsit, lésion qu'accompagne une adénopathie satellite. Le reste de l'examen somatique ne révèle rien de particulier. La formule sanguine montre 9000 leucocytes, avec $67 \%$ de segmentés, $4 \%$ de non segmentés, $11 \%$ de monocytes et $8 \%$ de lymphocytes. La VS est de $25 \mathrm{~mm}$ à la première heure. Les tests hépatiques, les urines et la radiographie pulmonaire sont sans partic ılarité, de même que les sérologies virales classiques. Après avoir rapproché ce cas des trois premiers, la malade est mise s ous doxycycline $200 \mathrm{mg}$ /jour, ce qui amène une défervescence dès le deuxième jour de traitement. Elle sera complètement rétablie une semaine plus tard.

Cas $n^{\circ}$ 5. M. C..., une jeune femme de 34 ans, en bonne santé habituelle, est hospitalisée d'urgence le 16 juillet 1985 dans le survice de médecine interne de l'Hôpital de La Chaux-deFonds, pour un état fébrile persistant uss cié à une éruption cutanée. L’affection a débuté brutalement le 7 juillet 1985 par une fièvre à $38,6^{\circ} \mathrm{C}$ ass sciée à de très importantes myalgies des membres inférieurs. Le 11 juillet, des mac.les rosécs a| l araissent sur les chevilles. Mme M. C... présente, de plus, une odinophagie et une ctice tol. $\mathrm{x}$ sèch de frissons. Le 12 juillet, le médecin Iraitant intr dui un traitement de salicylés et de Pénicil-

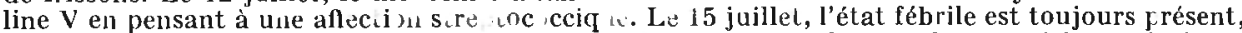
les myalgies ont encore augmeilé d'intusi. 
la musculature axiale. L'éruption s'est généralisée à l'ensemble du corps à l'exception du visage. L'hospitalisation est demandée.

A l'admission, la patiente est abattue, pâle, fébrile à $40^{\circ} \mathrm{C}$. On est d'emblée frappé par la présence d'un exanthème maculopapuleux, érythémateux, non prurigineux, particulièrement intense sur les fesses et les quatre membres. Plusieurs lésions sont observées sur les paumes des mains et les plantes des pieds. Malgré une recherche minutieuse, on ne découvre pas de "tache noire ". Mme M. C... présente une tachycardie sinusale à $136 /$ minute, une tachypnée à $26 /$ minute, associée à un sentiment de dyspnée. La tension artérielle est pincée à 100/80. Le reste du status est dans les limites de la norme, si l'on excepte la découverte d'un écoulement vaginal verdâtre dans lequel on mettra en évidence de nombreux Trichomonas vaginalis. Ia vitesse de sédimentation est de $121 \mathrm{~mm}$ à la première heure. Il n'y a pas d'anémie ni de thrombopénie, les leucocytes sont à 12400 avec une déviation gauche (non segmentés $18 \%$, segmentés $70 \%$, monocytes $6 \%$, lymphocytes $6 \%$ ). Le sodium plasmatique est à $140 \mathrm{mmol} / 1$, le potassium à $3,7 \mathrm{mmol} / 1$, les $\mathrm{GOT}$ sont augmentées à $949 \mathrm{nkat} / 1$, la phosphatase alcaline à 6,0 nkat/1 et les GGT à 4011 nkat/l. Les autres examens de laboratoire sont normaux, en particulier les GPT, la bilirubine et la créatinine plasmatiques. La radiographie du thorax et l'ECG sont également sans anomalie notable. On

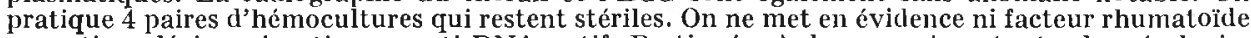
ou anti-nucléaire, ni anticorps anti-DNA natif. Pratiquées à deux reprises, toutes les sérologies virales, bactériennes ou parasitaires correspondant à une maladie exanthématique restent négatives. Les sérologies contre Coxiella burneti (IFAT) et $R$. mooseri (IFAT) ainsi que la réaction de Weil-Felix ne donnent pas non plus de résultat significatif. La sérologie contre $R$. conorii (IFAT) s'avère en revanche positive à $1 / 80$ (IgM et IgG) dans deux laboratoires différents. Lorsqu'on pose enfìn le diagnostic de FBM, au $20^{\circ}$ jour de la maladie, la fièvre commence à décrołtre rapidement et l'éruption a pratiquement disparu. On s'abstiendra finalement de toute antibiothérapie complémentaire devant cette évolution favorable.

\section{Discussion}

\section{CLINIQUE}

Le tableau clinique de la FBM est bien caractérisé, comme nous l'avons vu précédemment. Dans notre série, les quatre premiers malades ont présenté la même évolution classique avec une phase prodromale suivie d'une phase éruptive. On a observé chez chacun d'eux un état fébrile, accompagné de céphalées, de myalgies, d'arthralgies et d'une tache noire de Pieri précédant l'apparition d'un exanthème maculopapulaire généralisé. Ce dernier peut éventuellement détourner l'attention du médecin vers d'autres maladies éruptives fébriles, en particulier chez l'enfant, comme dans nos cas 2 et 3 . Lorsque la tache noire fait défaut ( $\operatorname{cas}^{\circ} 5$ ), la présence de maculopapules érythémateuses sur les paumes des mains et les plantes des pieds permet alors d'orienter le diagnostic vers une FBM. En effet, classiquement, seules les rickettsioses, les viroses à Coxsackies et la syphilis secondaire sont accompagnées de telles lésions.

Tous nos cas ont eu une évolution favorable, avec ou sans traitement. Des complications ont cependant été notées chez deux malades. La patiente $n^{0} 4$ a certainement présenté une atteinte encéphalitique (état confusionnel, myoclonies du membre supérieur droit et de la mâchoire) bien que celle-ci n'ait pas été confirmée par une ponction lombaire ou un EEG. De son côté, Mme M. C... devait développer une atteinte hépatique infraclinique de type plutôt cholestatique avec une augmentation de la phosphatase alcaline à 1,5 fois la norme et des GGT à 4 fois la norme. On notera encore chez cette patiente l'aspect particulièrement invalidant des myalgies, responsables d'une véritable pseudoparalysie antalgique des membres inférieurs entraînant un état grabataire pendant plus d'une semaine. 


\section{EPIDÉMIOLOGIE}

Bien qu'il ait été absent lorsque les cas nos 2,3 et 4 se sont déclarés, le chien de la patiente $n^{\circ} 1$ représente le trait d'union épidémiologique liant ces quatre malades. Il s'agissait d'un Schnautzer à poils abondants originaire du Tennessee, région des États-Unis où l'on trouve des tiques de l'espèce Dermacentor andersoni, vectrices de $R$. rickettsi, l'agent de la Fièvre pourprée des Montagnes Rocheuses. Il avait accompagné ses maîtres en Suisse en 1974. Depuis, il s'était souvent rendu avec eux dans le midi de la France, entre Cannes et la Camargue, ainsi qu'en Toscane en 1976. Ces régions de vacances sont des foyers bien connus de FBM. En effet, on y trouve en abondance la tique $R h$. sanguineus, vecteur de $R$. conorii. Si l'on excepte un épisode d'arthralgies et de faiblesse marquée en 1977, ce chien avait toujours été en excellente santé, bien qu'il héberge souvent des tiques malgré le port régulier de colliers acaricides. Durant l'arrière-automne 1980, après la maladie de la patiente $n^{\circ} 1$, il avait été examiné soigneusement à la recherche de tiques mais sans succès. On ne devait pas en trouver davantage dans l'appartement de ses maîtres. En revanche, une nouvelle inspection de ce logement, faisarit suite aux cas nos 2 et 3 , se solda par la découverte de centaines de nymphes, non seulement sur le chien, notamment entre les doigts des pattes, mais également dans son panier et les replis de sa couverture, dans les meubles et surtout dans le crépi des murs, à une hauteur de 1,5 à $2 \mathrm{~m}$. Plusieurs cadavres de femelles entourées d'œufs et de larves fraîchement écloses étaient également observés sous une valise, sous les meubles et même sous le matelas d'un lit. Ces tiques appartenaient toutes à l'espèce $R h$. sanguineus. Les tests d'hémolymphe (5), pratiqués sur certaines d'entre elles, révélèrent qu'elles étaient porteuses de Rickettsies (tableau I), appartenant à l'espèce $R$. conorii, comme devaient le démontrer Peter et al. (17). Sans aucun doute possible, ces tiques avaient été ramenées dans l'appartement genevois par le chien, au retour d'un voyage dans le sud de l'Europe. $R h$. sanguineus est en effet incapable de subsister dans la nature sous notre climat. Les chiens sont les hôtes préférentiels de ces tiques qui n'hésitent pourtant pas à s'attaquer à l'homme. Rappelons à ce sujet que la patiente no 4 devait tomber malade en 1981 , peu après le départ du Schnautzer, hébergé chez elle quelques jours. Trois $R$ h. sanguincus importés par cet animal furent d'ailieurs découverts dans son appartement (2).

TABlenU I. - Présence de Rickettsies dans l'hémolymphe de $R$. sanguineus récoltés dans un appartement de la région genevoise.

\begin{tabular}{lcc}
\hline $\begin{array}{c}\text { Stade } \\
\text { évolutif }\end{array}$ & $\begin{array}{c}\text { Présence } \\
\text { de Rickettsies }\end{array}$ & $\%$ d'infection \\
\hline 11 mâles & 5 & $46 \%$ \\
9 femelles & 5 & $56 \%$ \\
38 nymphes & 4 & $11 \%$ \\
\hline Totaux : 58 & 14 & $24 \%$ \\
\hline
\end{tabular}


Ces foyers genevois et neuchâtelois furent désinfectés à deux reprises, à un mois d'intervalle, par l'intermédiaire d'une entreprise spécialisée. Depuis, plus aucune tique n'a été obser vée et plus aucun cas de FBM ne s'est déclaré dans cette famille.

L'enquête épidémiologique menée à propos du 5 e cas devait s'avérer tout aussi difficile. Dans un premier temps et bien que Mme M. C... ait été interrogée à plusieurs reprises durant son hospitalisation, aucune explication satisfaisante ne pouvait être donnée sur la manière dont elle avait contracté cette FBM. La patiente n'avait plus quitté la Suisse depuis de nombreux mois. Le chien et le chat dont elle était propriétaire n'avaient jamais été emmenés à l'étranger. Aucune tique ne put d'ailleurs être découverte dans leur pelage, pas plus que dans l'appartement où ils résidaient. Mme M. C... n'avait eu aucun contact avec les autres animaux domestiques vivant dans la maison. Ce n'est que quelques jours avant sa sortie de l'hôpital qu'elle nous apprit s'être rendue chez sa sœur, environ 15 jours avant le début de sa maladie. Celle-ci venait de rentrer d'Ardèche où son chien l'avait accompagnée. Il avait porté des tiques à plusieurs reprises durant le voyage et on en avait encore découvert en Suisse. Mme M. C... avait longtemps joué avec lui lors de sa visite et avait probablement été piquée par une tique à cette occasion. Il ne nous fut malheureusement pas possible d'examiner cet animal, ni d'inspecter la maison où il vivait. Cependant, la sœur de Mme M. C..., bien informée des risques encourus à cohabiter avec des Rhipicéphales, en rechercha minutieusement sur son chien et dans son logement, sans succès. Elle nous promit pourtant de rester attentive à ce problème et de nous adresser toutes les tiques qu'elle pourrait trouver. A ce jour, nous n'avons pas reçu de ses nouvelles.

\section{Conclusions}

Les rickettsioses à tiques étant rares dans notre pays, le clinicien oublie trop facilement d'évoquer leurs diagnostics. En face d'un tableau associant fièvre, algies diffuses et exanthème étendu, il devrait pourtant penser à la FBM, même chez une personne n'ayant jamais quitté la Suisse, et rechercher la présence d'une tache noire de Pieri ou de maculopapules des paumes des mains et des plantes des pieds.

Si le diagnostic de FBM est posé, il faut le confirmer sérologiquement et traiter le malade. Toutefois, la tâche du médecin ne s'arrête pas là ; il doit mener une enquête épidémiologique minutieuse, avec l'aide des Services de Santẻ Publique, afin de déterminer les conditions dans lesquelles le patient a été contaminé et chercher à prévenir, ainsi, l'apparition d'autres cas parmi ses proches. On se renseignera sur les derniers voyages du malade à l'étranger, sur la présence d'un chien dans son entourage, sur la découverte de tiques dans le pelage de cet animal ou dans l'appartement qu'il occupe. Ces questions portant sur des événements apparemment anodins pour le patient, les résultats sont rarement immédiats. Cette anamnèse devra donc être répétée auprès du patient bien sûr, mais également auprès de ses parents, voisins et amis. Par le biais de cette véritable enquête poli- 
cière on pourra éventuellement découvrir des Rhipicéphales précocement et les détruire avant qu'ils ne se développent en un véritable foyer domestique ou qu'ils ne contaminent d'autres personnes.

\section{BIBLIOGRAPHIE}

1. Aeschlimans A., Buttiker W. Importations de Tiques en Suisse (Acarina: Ixodoidea). Bull. Soc. Entomol. Suisse, 1975, 48, 69-75.

2. Aeschlimann A., Chatelanat P., Bowesidjaou J., Schneeberger S. : Importierung von "exotischen "Zecken und Rickettsien in die Schweiz: Schweiz. Tropenmedizin, Parasitologie/ Joseph Boch (H25 g). Ed. P. Lang, Frankfurt a. M., 1984, p. 377.

3. Best J. M. J., ButT K. M., Rohrbach J. A. : Occurrence of Rhipicephalus sanguineus in London. Vet. Rec., 1969, 85, 633.

4. Bourgeade A., Jean-Pastor M. J., Raoult D. : Notes sur la Fièvre Boutonneuse Méditerranéenne (à propos de 40 cas récents à Marseille). Bull. Soc. Pathol. Exot., 1979, 308-314.

5. Burgdorfer W.: Hemolymph test. A technique for detection of Rickettsiae in ticks. Am. J. Trop. Med. Hyg., 1970, 19, 1010-1014.

6. Cloppet H., Quenin P., Vincent P. : Valeur actuelle de l'immunofluorescence indirecte et de la réaction de fixation du complément dans le diagnostic des Rickettsioses. Med. Mal. Infect., 1980, 10, 307-310.

7. Dezest G. : Les Fièvres Boutonneuses du Cameroun sont graves. Bull. Soc. Pathol. Exot., 1953, XLVI, 353-359.

8. Edinger E. : Serological diagnosis of Mediterranean Spotted Fever. Ann. Microbiol., 1979, $130 \mathrm{~A}, 203-211$.

9. Enlinger E., Navarro P. : Les Rickettsioses : une morbidité du tourisme. Sem. Hop. Paris, $1983,59,2053-2054$.

10. Gotнe R. : Zur Vorkommen von Rhipicephalus sanguineus (Latreille 1806) in Deutschland. Tropenmed. Parasitol., 1968, 19, 305-307.

11. Guardia J., Martinez-Vasquez J. M., Moragas A., Rey C., Vilaseca J., Tornos J., Beltran M., Bacardi R. : The liver in Boutonneuse Fever. Gut, 1974, 15, 549-551.

12. Hackman W. : Fästingen Rhipicephalus sanguineus funnen i Finland (Acarina, Ixodoidae). Mem. Soc. Fauna. Flora. Fenn., 1977, 53, 116.

13. Lefebvre J. C., Favier G., Dellamonica P., Vandekerkove M. : Diagnostic de la Fièvre Boutonneuse Méditerranéenne par Immunofluorescence indirecte. Nouv. Presse Med., $1979,8,1431$.

14. Mansueto S., Vitale G., Bentivegna M., Tringali G., Di Leo R. : Persistence of antibodies to Rickettsia conori after an acute attack of Boutonneuse Fever. J. Infect. Dis., 1985, 151, 377.

15. Moraga F. A., Martinez-Roig A., Alonso J. L., Boronat M., Domingo F. : Boutonneuse Fever. Arch. Dis. Child, 1982, 57, 149-151.

16. Olmer D., Olmer J. : Épidémie de Rickettsioses sur le littoral méditerranéen : la Fièvre Boutonneuse. Rev. Pathol. Gen. Comp., 1956, 56, 80-92.

17. Peter O., Burgdorfer W., Aeschlimann A., Chatelanat P. : Rickettsia conorii isolated from Rhipicephalus sanguineus introduced into Switzerland on a pet dog. Z. Parasitenkd., $1984,70,265-270$.

18. Raoult D., Weiller P. J., Esterni J. P., Garnier J. M., Gallais H., Casanova P. : La Fièvre Boutonneuse Méditerranéenne. Concours Med., 1983, 105, 2933-2940.

19. Raoult D., Jean-Pastor M. J., Xeridat B., Garnier J. M., Weiller P. J., Gargin G., Privat Y., Gallais H., Casanova P. : La Fièvre Boutonneuse Méditerranéenne : à propos de 154 cas récents. Ann. Dermatol. Venereol., 1983, 110, 909-914.

20. Raybaut A., Tartaroli M. A. : La méningite dans la Fièvre Boutonneuse Méditerranéenne. Marseille Med., 1965, 4, 317-319.

21. Santoni A., Bertrand A. : La Fièvre Boutonneuse Méditerranéenne. Med. Hyg., 1985, $1602,898-902$.

22. Vachon F., Fouqueray B., Balestie B., Katlama C. : Syndrome mononucléosique au cours d'une infection à Rickettsia conori. Presse Med., 1984, 13, 1961-1962.

23. Winding O., Hanarov N. : Accidental introduction into Denmark from Sudan of the Renuel tick (Rhipicephalus sanguineus Latr. 1806). Nord. Vet. Med., 1968, 20, 284-285. 